SHORT REPORT

\title{
Initial DWI and ADC imaging may predict outcome in acute disseminated encephalomyelitis: report of two cases of brain stem encephalitis
}

\author{
H Axer, A Ragoschke-Schumm, J Böttcher, C Fitzek, O W Witte, S Isenmann
}

J Neurol Neurosurg Psychiatry 2005;76:996-998. doi: 10.1136/jnnp.2004.045500

Two young patients with acute disseminated encephalomyelitis (ADEM) of the brain stem are described. In spite of similar lesion sites in the brain stem, reaching from the upper medulla to the mesencephalon, the outcomes of the patients were very different: one made a full clinical recovery within three weeks while the other remained in a locked-in state more than a year after the disease episode. Both patients also differed in magnetic resonance imaging (MRI) findings on admission. The patient who remained in a locked-in state had pathological diffusion weighted imaging (DWI) scans and decreased apparent diffusion coefficient maps initially, with severe tissue destruction on follow up computed tomography, while the patient who recovered fully showed initially increased apparent diffusion coefficient values and almost complete resolution of MRI changes on follow up. Thus a comparison of these two cases may indicate differences in the underlying pathology in ADEM (vasogenic $v$ cytotoxic oedema) that may be crucial for estimating tissue damage and clinical outcome.

A cute disseminated encephalomyelitis ${ }^{1}$ (ADEM) is a monophasic inflammatory condition of the central nervous system characterised by acute demyelination that may follow infection or vaccination. Prognosis is often favourable. However, severe cases may be lethal or result in persisting disability. Reliable indices for predicting prognosis in individual cases have not been established.

We present two cases of ADEM with similar initial brain stem lesions but a different course. We suggest that magnetic resonance imaging (MRI) with diffusion weighted imaging (DWI) and apparent diffusion coefficient (ADC) mapping at the time of admission may predict outcome. Diffusion weighted MRI measures the motion of water molecules, thus documenting water diffusion in the tissue. The ADC is a value calculated from these data and represents the rotationally invariant measurement of total diffusion within the tissue. ${ }^{23}$

\section{CASE REPORTS}

\section{Case 1}

A 29 year old male carpenter presented with severe headache shortly after an afebrile upper respiratory tract infection. Neurological examination was normal except for slight meningism. Initial brain computed tomography was normal. Lumbar puncture showed 400 cells/ $\mu$ l. Antibiotic treatment was started with ceftriaxon and ampicillin. The antiviral agent acyclovir was given as well. The following day, the patient became drowsy, developed vertical nystagmus and vomiting, and had generalised epileptic seizures requiring orotracheal intubation and treatment with valproate. Brain
MRI showed symmetrical hyperintensities in the upper medulla, pons, cerebellar peduncles, midbrain, and internal capsules on both sides in a fluid attenuated inversion recovery (FLAIR) sequence (fig lA). Conventional T2 imaging showed hyperintense lesions mainly located in the white matter of the brain stem, reaching from the upper medulla to the mesencephalon, cerebellar peduncles, and internal capsules. Conventional Tl imaging was normal. No contrast enhancement was found. Vascular morphology was normal on magnetic resonance angiography. DWI signal intensities (fig 1B) were normal, and ADC maps (fig 1C) were increased (quantitative ADC with region of interest (ROI) area $20 \mathrm{~mm}^{2}$, mean (SD): centrum semiovale, 6.32 $(0.48) \times 10^{-4} \mathrm{~mm}^{2} / \mathrm{s}$; mesencephalon, $8.45(1.40) \times 10^{-4} \mathrm{~mm}^{2} / \mathrm{s}$; pons, $\left.7.91(0.79) \times 10^{-4} \mathrm{~mm}^{2} / \mathrm{s}\right)$.

CSF now contained 886 cells/ $\mu$ l. Intrathecal $\operatorname{IgG}$ and $\operatorname{IgM}$ synthesis was present, but oligoclonal bands were negative. Destruction of CNS parenchyma, with predominant demyelination, was indicated by increased S-100 (15.8 $\mu \mathrm{g} / \mathrm{l}$; normal $<3.3 \mu \mathrm{g} / \mathrm{l}$ ) and myelin basic protein (MBP) $(31.6 \mathrm{ng} / \mathrm{ml}$; normal $<5 \mathrm{ng} / \mathrm{ml}$ ). Methylprednisolone was added for seven days. Serological tests for tick borne encephalitis, Lyme borreliosis, listeriosis, cytomegalovirus, herpes simplex virus, varicella-zoster virus, Epstein-Barr virus, brucellosis, mycoplasma pneumoniae, and other organisms were all negative, as were screening for paraneoplastic autoantibodies and autoimmune serology. Although the CSF cell count decreased to $24 / \mu$ l on day 7 , extubation failed, necessitating tracheotomy on day 10. After withdrawal of sedation, the patient breathed spontaneously on day 15 and made a complete clinical recovery. On day 18, MRI showed clearly decreased pathological changes (fig 1D). At this time, residual lesions were confined to the middle cerebellar peduncles. In a neurological examination three months later the patient had no neurological symptoms.

\section{Case 2}

A 21 year old woman was found comatose after suffering from headache and vertigo the evening before. Two weeks previously she had had diarrhoea. Initial brain MRI showed symmetrical hyperintensities in the upper medulla, pons, midbrain, and right thalamus on FLAIR (fig $1 \mathrm{E}$ ) and hyperintense lesions in the white matter reaching from the medulla to the mesencephalon on conventional T2 weighted scans. Vascular morphology was normal on magnetic resonance angiography. There was slight contrast enhancement at the lesion rim, and some blood was detected in the pons using susceptibility weighted T2. In contrast to case 1 , DWI showed pathological high signal intensities (fig l F), and ADC maps (fig $1 \mathrm{G}$ ) were pathologically low (quantitative

Abbreviations: $A D C$, apparent diffusion coefficient; $A D E M$, acute disseminated encephalomyelitis; DWI, diffusion weighted imaging 

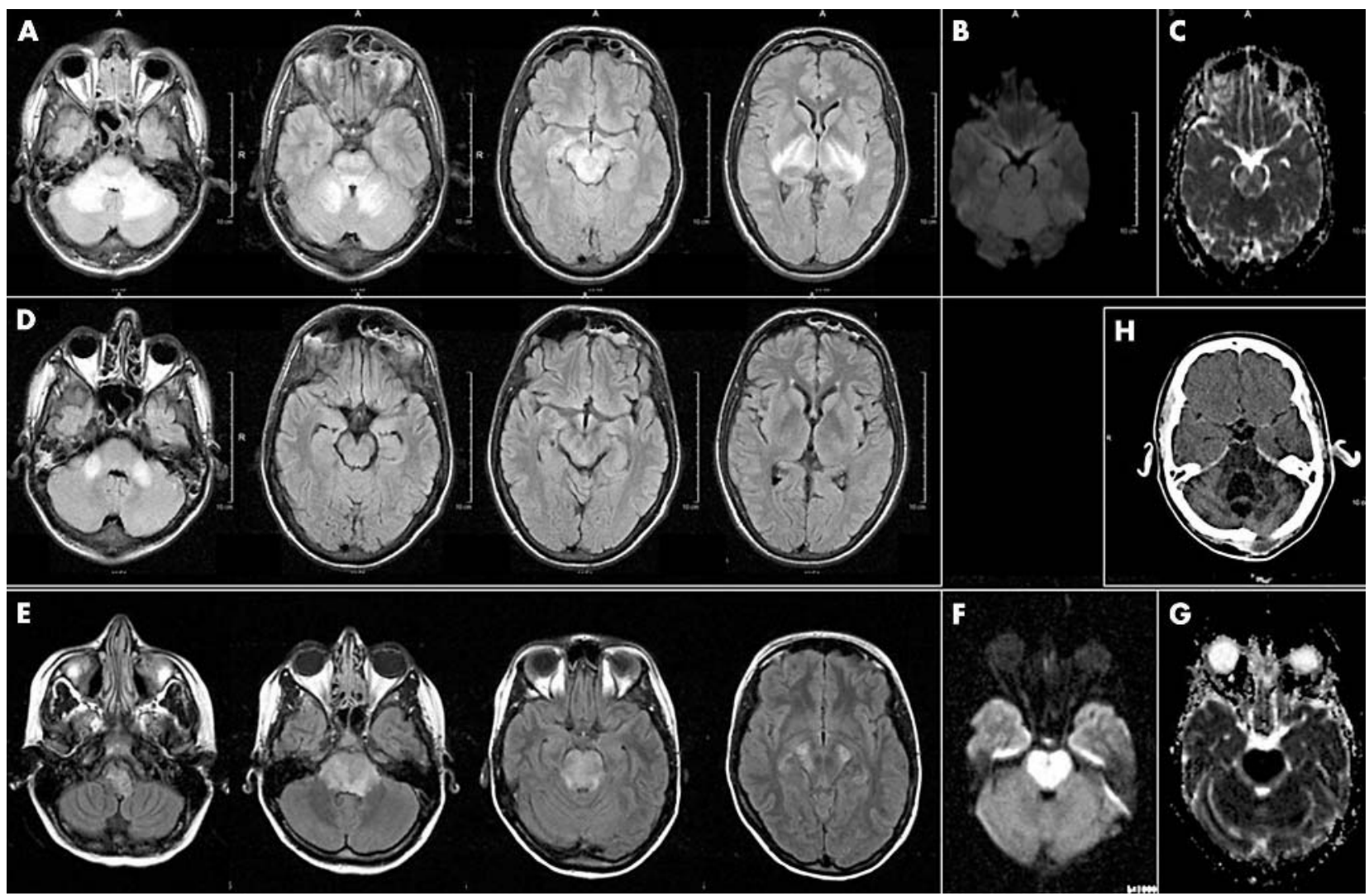

Figure 1 (A) Fluid attenuated inversion recovery (FLAIR) images of case 1 at onset of symptoms, with normal diffusion weighted imaging (DWI) signal intensities (B) and increased apparent diffusion coefficient (ADC) maps (C). (D) FLAIR images of case 1 on day 18, showing almost full recovery of tissue with enhanced signal only in the cerebellar peduncles. (E) FLAIR images of case 2 shows similar lesions as case 1, but increased DWI signal (F) and pathologically low ADC maps (G). (H) Cranial computed tomography one year after onset of symptoms, showing the tissue defect.

ADC with ROI area $20 \mathrm{~mm}^{2}$ : centrum semiovale, 7.66 $(0.87) \times 10^{-4} \mathrm{~mm}^{2} / \mathrm{s}$; mesencephalon, $4.10(0.41) \times 10^{-4} \mathrm{~mm}^{2} / \mathrm{s}$; pons, $\left.4.26(0.58) \times 10^{-4} \mathrm{~mm}^{2} / \mathrm{s}\right)$. Lumbar puncture showed 117 cells $/ \mu$ l. Serological tests, as detailed in case 1, were negative. Antibiotic, antiviral, and anti-inflammory treatment was started (acyclovir, ceftriaxon, ampicillin, and methylprednisolone). The following day, MRI showed an increase in the lesion size, including the midbrain and right thalamus. Plasmapheresis was initiated, followed by intravenous cyclophosphamide. After tracheotomy and termination of sedation, no improvement was seen. Weaning was difficult. The patient remained comatose with tetraplegia. During more than eight months of rehabilitation, she remained in a locked-in state, able to communicate through vertical movements of the right eye only. Figure $1 \mathrm{H}$ shows brain CT one year after disease onset, with a persistent lesion.

\section{DISCUSSION}

In both cases the diagnosis of brain stem ADEM was based on the clinical syndrome and course, the imaging results, and negative serology. Acute monophasic symmetrical brain stem lesions may be caused by inflammation, central pontine myelinolysis, tumour, bleeding, or ischaemia. Increased CSF white cell counts classified our cases as inflammatory. Brain stem encephalitis may be caused by infectious agents (for example, mycoplasma, listeria, herpes simplex virus, cytomegalovirus, Epstein-Barr virus), or it may occur as a paraneoplastic syndrome. These differential diagnoses were excluded in our patients.

In both cases, encephalitis was triggered by a preceding infection. The lesion sites were similar in that medulla, pons, midbrain, and diencephalon were involved, and inflammation extended along fibre tracts to the cerebellar peduncles and the internal capsules. The main differences were found in DWI and ADC maps. Case l showed no pathological signal intensities on DWI, and increased ADC maps; the patient recovered completely within three weeks. In contrast, case 2 showed increased signal intensities on DWI, and pathologically decreased ADC maps. Despite early treatment, outcome was poor. Additional imaging findings in this patient included contrast enhancement and haemorrhage in the pons, which may also be signs of the severity of the process and may indicate a poor neurological prognosis.

From these two cases, it appears that the course and outcome can be assessed on admission using MRI including DWI and ADC maps. Sener ${ }^{4}$ described diffusion MRI findings in herpes simplex virus encephalitis and found that pathological ADC maps were associated with a poor outcome. Vasogenic oedema can lead to pathological high signal intensities in ADC maps, because of T2 shining-through effects even in DWI sequences, but this may be reversible. In contrast, cytotoxic oedema is characterised by low ADC values and may indicate tissue necrosis. However, reduced ADC values cannot on their own be attributed to irreversible tissue necrosis in every instance. Reversibility of reduced ADC values has been demonstrated in hypoglycaemia ${ }^{5}$ and in several patients with heterogeneous diagnoses. ${ }^{6}$ In brain ischaemia, a decrease in ADC is generally attributed to cell swelling caused by failure of the transmembrane $\mathrm{Na}^{+} / \mathrm{K}^{+}$ pump, leading to impairment of cell membrane potential and alteration of ion homeostasis. ${ }^{5}$ Recovery of ADC changes after normalisation of the cerebral circulation shows that a 
decrease in ADC may indicate a functional disturbance of the cells that is potentially reversible in certain instances. ${ }^{7}$

ADC changes in CNS inflammation-for example, in demyelinating lesions-have been reported, but the underlying pathophysiological processes and their significance are still not fully understood. ${ }^{8}$ Moreover, other factors such as cellular infiltrates may also account for decreased ADC values in inflammatory conditions. Thus the findings in the two patients reported here may not be valid for a larger population and a follow up evaluation of more cases is required.

Nevertheless, the ADC reduction in the second case clearly preceded the irreversible brain stem lesion. Reports on DWI in brain stem encephalitis or ADEM are rare. Two papers have described changes in DWI in $\mathrm{ADEM}^{8}{ }^{9}$ that may also occur in other CNS inflammatory diseases. ${ }^{10-12}$ Harada et $a l^{13}$ reported two children-one with ADEM and one with acute necrotising encephalopathy (ANE). They found decreased ADC values in the ANE case and increased values in the ADEM case. They stated that the diffusion of brain water might be an index of reversibility of neuronal impairment. Thus these observations are similar to our cases. The imaging features suggest vasogenic oedema in case 1 , in contrast to cytotoxic oedema in case 2 . The visualisation of the probable underlying pathology may be an indicator of outcome, while electrophysiology for estimation of the prognosis and severity of brain stem lesions remains controversial. ${ }^{14}$

\section{Authors' affiliations}

H Axer, A Ragoschke-Schumm, O W Witte, S Isenmann, Department of Neurology, Friedrich Schiller University Jena, Jena, Germany

J Böttcher, C Fitzek, Department of Diagnostic and Interventional Radiology, Friedrich Schiller University Jena

Competing interests: none declared
Correspondence to: PD Dr med Hubertus Axer, Department of Neurology, Friedrich-Schiller-University Jena, Erlanger Allee 101, D07747 Jena, Germany; Hubertus.Axer@med.uni-jena.de

Received 11 May 2004

In revised form 28 October 2004

Accepted 29 October 2004

\section{REFERENCES}

1 Schwarz S, Mohr A, Knauth M, et al. Acute disseminated encephalomyelitis: a follow-up study of 40 adult patients. Neurology 2001;56:1257-60.

2 Fitzek C, Mentzel HJ, Fitzek S, et al. Echoplanar diffusion-weighted MRI with intravenous gadolinium-DTPA. Neuroradiology 2003:45:592-7.

3 Bilgili $Y$, Unal B. Effect of region of interest on interobserver variance in apparent diffusion coefficient measures. Am J Roentgenol 2004;25:108-11. 4 Sener RN. Herpes simplex encephalitis: diffusion MR imaging findings. Comput Med lmaging Graph $2001 ; 25: 391-7$.

5 Hasegawa Y, Formato J, Latour LL, et al. Severe transient hypoglycemia causes reversible change in the apparent diffusion coefficient of water. Stroke 1996;27:1648-55.

6 Grant PE, He J, Halpern EF, et al. Frequency and clinical context of decreased apparent diffusion coefficient reversal in the human brain. Radiology 2001;221:43-50.

7 Fiehler J, Knudsen K, Kucinski T, et al. Predictors of apparent diffusion coefficient normalization in stroke patients. Stroke 2004;35:415-19

8 Bernarding J, Braun J, Koennecke HC. Diffusion- and perfusion-weighted MR imaging in a patient with acute demyelinating encephalomyelitis (ADEM). $J$ Magn Reson Imaging 2002;15:96-100.

9 Küker W, Ruff J, Gaertner S, et al. Modern MRI tools for the characterization of acute demyelinating lesions: value of chemical shift and diffusion-weighted imaging. Neuroradiology 2004;46:421-6.

10 Tsuchiya K, Katase S, Yoshino A, et al. Diffusion-weighted MR imaging of encephalitis. AJR Am J Roentgenol 1999;173:1097-9.

11 McCabe K, Tyler K, Tanabe J. Diffusion-weighted MRI abnormalities as a clue to the diagnosis of herpes simplex encephalitis. Neurology 2003;61:1015-16.

12 Agid R, Ducreux D, Halliday WC, et al. MR Diffusion-weighted imaging in a case of West Nile virus encephalitis. Neurology 2003;61:1821-3.

13 Harada M, Hisaoka S, Mori K, et al. Differences in water diffusion and lactate production in two different types of postinfectious encephalopathy. J Magn Reson Imaging 2000;1 1:559-63.

14 Gütling E, Isenmann S, Wichmann W. Electrophysiology in the locked-insyndrome. Neurology 1996;46:1092-101. 